\title{
Simulating Strongly Correlated Quantum Systems with Tree Tensor Networks
}

\author{
V. Murg and F. Verstraete \\ Fakultät für Physik, Universität Wien, Boltzmanngasse 3, A-1090 Vienna, Austria \\ Ö. Legeze⿴囗十 and R. M. Noack \\ Fachbereich Physik, Philipps-Universität Marburg, 35032 Marburg, Germany
}

(Dated: October 23, 2018)

\begin{abstract}
We present a tree-tensor-network-based method to study strongly correlated systems with nonlocal interactions in higher dimensions. Although the momentum-space and quantum-chemistry versions of the density matrix renormalization group (DMRG) method have long been applied to such systems, the spatial topology of DMRG-based methods allows efficient optimizations to be carried out with respect to one spatial dimension only. Extending the matrix-product-state picture, we formulate a more general approach by allowing the local sites to be coupled to more than two neighboring auxiliary subspaces. Following Shi. et. al. [Phys. Rev. A, 74, 022320 (2006)], we treat a tree-like network ansatz with arbitrary coordination number $z$, where the $z=2$ case corresponds to the one-dimensional scheme. For this ansatz, the long-range correlation deviates from the meanfield value polynomially with distance, in contrast to the matrix-product ansatz, which deviates exponentially. The computational cost of the tree-tensor-network method is significantly smaller than that of previous DMRG-based attempts, which renormalize several blocks into a single block. In addition, we investigate the effect of unitary transformations on the local basis states and present a method for optimizing such transformations. For the 1-d interacting spinless fermion model, the optimized transformation interpolates smoothly between real space and momentum space. Calculations carried out on small quantum chemical systems support our approach.
\end{abstract}

\section{INTRODUCTION}

Understanding and simulating strongly correlated systems has long been a major challenge in theoretical physics and in theoretical chemistry. In the past two decades, the density-matrix renormalization group (DMRG) method has been applied effectively to study problems in these fields $\frac{1,2}{2}$ In particular, it has been widely used to study fermionic and spin-chain problems in one dimension for models with both local and long-range interactions. Application to systems with long-range interactions gained impetus when the method was reformulated to treat models defined in mo-

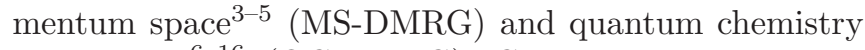
calculations $-\underline{6}$-16 (QC-DMRG). Common characteristics of these approaches are that a higher dimensional system is mapped to a one-dimensional chain and that variational approximations to the eigenstates are obtained by an iterative diagonalization procedure. Introduction of various quantum information entropies ${ }^{5,17-19}$ and the reformulation of the problem in terms of matrix product states $^{20}-22$ (MPS) has clarified the mathematical underpinnings of the method.

Recently, extensions to two dimensions based on the controlled manipulation of entanglement between subsystems has led to alternate methods that can be viewed as generalizations of matrix-product-state-based methods $\stackrel{23}{=}$ These methods include projected entangled pair states $20,24-26$ (PEPS), the multiscale-entanglementrenormalization ansatz ${ }^{27}$ (MERA), and correlator product states 28 (CPS) or complete-graph tensor network states29 (CGTN). The PEPS and MERA methods have already shown considerable promise for frustrated and fermionic problems; they do not suffer from the fermion sign problem that appears in quantum Monte Carlo simulations $\underline{30,31}$ These new methods, however, have primarily been restricted to the treatment of local Hamiltonians. Thus, developing effective algorithms to treat higher dimensional systems in which the interactions are nonlocal remains an important problem.

In order to efficiently treat quantum chemical systems, it has become evident in the past few years that methods must take into account more general spatial topology. It has been shown that quantum information entropy ${ }^{5.15}$

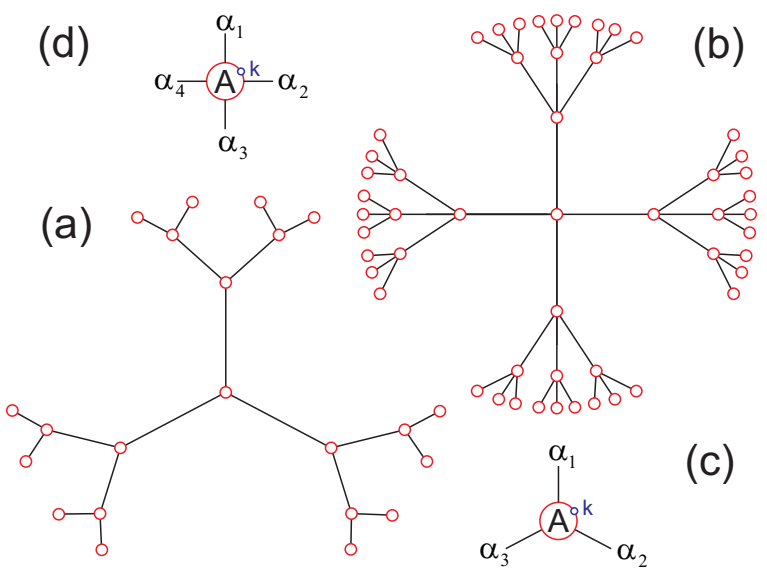

FIG. 1. (Color online) Tree tensor network with $z=3$ (a) and $z=4(\mathrm{~b})$. The structure of the tensors is shown in (c) and (d). The bonds indicate the virtual indices $\alpha_{1}, \ldots, \alpha_{z}$ and the circle the physical index $k$. 
can be used to determine the entanglement or quantum correlation among sites or orbitals in a pairwise way. Such a two-dimensional entanglement matrix leads to a picture of the topology of orbitals that corresponds to a multiply connected network. Two possible approaches to optimize computational efficiency are to work with an appropriate network-like structure that reflects the entanglement topology and to vary the single-particle basis 32 so that entanglement is reduced. For states based on a one-dimensional topology, the only possibility to take entanglement topology into account is to reorder the orbitals. The next step in generalizing the topology is to use a tree network. This was first pointed out in Ref. [33]. A tree network makes it possible to reduce the distance between highly entangled orbitals when they are multiply connected. In addition, the coordination number $z$ can be varied from orbital to orbital to adapt the variational state to a particular entanglement topology. For a tree network that is bipartite, it is possible to adapt methods and optimizations developed for matrix-product-statebased algorithms such as the DMRG ${ }^{34}$ to tensor-product states on the tree network. This is because the tree network method can be related to a generalized DMRG with $z$ blocks instead of two. In addition, since an effective Hamiltonian can be formed, real time evolution, like that done in t-DMRG ${ }^{18,35-37}$ and in PEPS25, can be carried out. Real time evolution could be used, for example, to calculate spectral functions for quantum chemical systems.

The use of more general spatial topologies is also potentially important for quantum impurity problems. In these systems, the impurity subsystem, which can consist of either a single site or a strongly correlated cluster, is coupled to a bath of free fermions. The classical method to treat quantum impurity problems numerically is the numerical renormalization group (NRG) ${ }^{38}$. The starting point of the NRG method is the mapping of the problem onto a one-dimensional semi-infinite lattice in which the first site (or set of sites) describes the impurity subsystem, and the remainder of the chain represents the logarithmically discretized conduction band. In the past few years, the NRG has been extended by dividing the chain into a system and an environment as in the DMRG ${ }^{39}$; the resulting method, the density-matrix numerical renormalization group (DM-NRG), has undergone significant development recently $40-44$ While these extensions have led to significant improvements, they nevertheless have focused primarily on optimizing algorithms based on a one-dimensional topology. In general, the conduction bands are entangled through the impurity subsystem only. Therefore, it is a natural choice to describe the problem as a tree-like structure in which the impurity subsystem at the center is surrounded by shells of the conduction bath. Such a tree-like topology has been utilized to treat the quantum impurity problem that occur with dynamic mean field theory (DMFT) $\underline{45,46}$

The first attempts to apply matrix-product states on tree networks were carried out by Otsuka 47 and by Fried- man et. al $\stackrel{48}{ }$, who calculated ground-state properties of the spin-1/2 XXZ and Heisenberg chains using the DMRG. Subsequently, Lepetit et. al $\frac{49}{}$ studied the halffilled Hubbard model on a Bethe lattice (also known as a Cayley tree). All of this work uses a Bethe lattice, whose characteristics are that the number of nearest neighbors at each node is $z$, i.e., the coordination number, and that closed loops do not occur. Since there is only one path between any pair of sites, a DMRG-based solution of the problem is possible. In this approach, however, $z$ systems blocks must be renormalized to a single block at each iteration step, leading to computational cost which increases exponentially with $z$, hindering systematic DMRG studies for large systems and/or large $z$; up to now only $z=3$ has been treated. In contrast, the tree tensor network (TTNS) methods we introduce here have a much lower computational cost because the topology consists of a single site and $z$ blocks, whereas the superblock configuration included $2 z$ blocks or $z+1$ in previous DMRG attempts. In this work, we present calculations on small systems in order to demonstrate the viability of the tree tensor network method and to compare its accuracy and efficiency with existing DMRG methods.

Another approach to take advantage of the benefits of the tree network was formulated in Ref. [50]. In this work, the tree tensor network is formed by placing physical sites on the boundary sites only. The remaining interior sites are virtual; they are only used to transfer entanglement up the tree. This tree tensor product state is designed to treat models in which sites contribute the same amount of entanglement, i.e, have the same value of single-site entropy.

In this work, we form a tree tensor network in which all sites in the tree represent physical sites and in which entanglement is transferred via the virtual bonds that connect the sites. Our motivation is to treat models in which physical sites have varying degrees of entanglement; positions closer to the center of the tree should be better suited to represent more entangled sites. An additional motivation is to take advantage of the property of the tree tensor network ansatz that the long-range correlations differ from the mean-field value polynomially with distance rather than exponentially with distance as for MPS. In our algorithmic approach to optimize the tree tensor network, we use tools similar to those used in Refs. [33] and [50], but optimize the network site-by-site as in the DMRG instead of performing an imaginary time evolution. In addition, we explicitly describe how to deal with fermions and long-range interactions.

The paper is organized as follows. In Sec. II we describe the theoretical background for the TTNS algorithm and the orbital optimization used during the iterative procedure. Sec. III is devoted to the analysis of the error in the ground-state energy for various spin and fermion models as a function of bond dimension for TTNS with different coordination numbers. These include the 2-d Heisenberg model, the 2-d spinless fermion model, the 1-d spinless fermion model in momentum 
space, and the Beryllium atom as an application in quantum chemistry. In the latter case, we compare the results to those from DMRG calculations. We conclude and discuss future prospects in Sec. IV]

\section{THEORETICAL BACKGROUND OF THE TREE-NETWORK APPROACH}

We approach the problem of finding the ground state of strongly correlated systems with long-range interactions using a TTNS ansatz of the form

$$
|\Psi\rangle=\sum_{k_{1}, \ldots, k_{M}} C_{k_{1} \ldots k_{M}}\left|k_{1}, \ldots, k_{M}\right\rangle
$$

Here the coefficients $C_{k_{1} \ldots k_{M}}$ describe a tree tensor network, i.e., they emerge from contractions of a set of tensors $\left\{A_{1}, \ldots, A_{M}\right\}$ according to a tree network, as shown in Fig. 1. We associate a tensor with $z+1$ indices,

$$
\left[A_{m}\right]_{\alpha_{1} \ldots \alpha_{z}}^{k}
$$

with each vertex $m$ of the network, that is, each tensor has $z$ virtual indices $\alpha_{1} \ldots \alpha_{z}$ of dimension $D$ and one physical index $k$ of dimension $d$, with $z$ being the coordination number of that site. The coefficients $C_{k_{1} \ldots k_{M}}$ are obtained by contracting the virtual indices of the tensors according to the scheme of a tree tensor network (see Fig. 11). The structure of the network can be arbitrary and the coordination number can vary from site to site. The only condition is that the network is bipartite, i.e., by cutting one bond, the network separates into two disjoint parts. In the special case $z=2$, the one-dimensional MPS-ansatz used in DMRG is recovered.

In a tensor network, entanglement is transferred via the virtual bonds that connect the sites. Thus, it is preferable to put strongly correlated sites close together, i.e., to minimize the number of bonds between them. Evidently, the diversity of arranging the sites in the network increases drastically with increasing coordination number $z$. Also, with a coordination number $z>2$ the number of virtual bonds required to connect two arbitrary sites scales logarithmically with the number of sites $M$, whereas the scaling is linear in $M$ for $z=2 \stackrel{33}{\underline{3}}$ This can be seen by considering a Cayley-tree of depth $\Delta$, as shown in Fig. 1. The number of sites in the tree is

$$
M=1+z \sum_{j=1}^{\Delta}(z-1)^{j-1}=\frac{z(z-1)^{\Delta}-2}{z-2}
$$

and thus, the maximal distance between two sites, $2 \Delta$, scales logarithmically with $M$ for $z>2$. This logarithmic scaling is fundamental because, with a MPS ansatz, the expectation value of a long-range correlation differs from the mean-field result only by a quantity that decays exponentially with the distance:

$$
\left\langle\tau_{n} \tau_{n+\Delta}\right\rangle-\left\langle\tau_{n}\right\rangle\left\langle\tau_{n+\Delta}\right\rangle \propto c^{-\Delta}
$$

With a TTNS ansatz, the logarithmic scaling counteracts this exponential decay, so that the difference from the mean-field result only scales polynomially with the distance.

The way to arrange the physical sites on the network is determined by the choice of the basis $\left|k_{1}, \ldots, k_{M}\right\rangle$. Obviously, the precision of the ansatz depends critically on the choice of the basis. For example, the noninteracting Fermi gas has a ground state that is a direct product in the momentum space representation. Such a state corresponds to a tree tensor network state with virtual dimension $D=1$ at all bonds. In position representation, however, a bond dimension that increases exponentially with the number of sites would be required. Thus, it will be favorable to optimize not only over the tensors in the coefficients $C_{k_{1} \ldots k_{M}}$, but also over the basis $\left|k_{1}, \ldots, k_{M}\right\rangle$.

In second quantization, $\left|k_{1}, \ldots, k_{M}\right\rangle$ denotes the basis in occupation number representation,

$$
\left|k_{1}, \ldots, k_{M}\right\rangle=\left(a_{1}^{\dagger}\right)^{k_{1}} \cdots\left(a_{M}^{\dagger}\right)^{k_{M}}|0\rangle
$$

and a basis transformation is obtained from the canonical transformation

$$
b_{j}^{\dagger}(U)=\sum_{r=1}^{M} U_{j r} a_{r}^{\dagger}
$$

defined by the $M \times M$ unitary matrix $U$. The whole variational ansatz then depends on two sets of parameters: the tensors $\left\{A_{1}, \ldots, A_{M}\right\}$ and the unitary $U$. The goal of the algorithm is to find the minimium of the energy with respect to these two sets of parameters, i.e., to calculate

$E=\min _{A_{1} \ldots A_{M} U}\left\langle\Psi\left(A_{1}, \ldots, A_{M}, U\right)|H| \Psi\left(A_{1}, \ldots, A_{M}, U\right)\right\rangle$.

The idea is to perform the optimizations over the parameter sets $\left\{A_{1}, \ldots, A_{M}\right\}$ and $U$ consecutively and repeatedly until convergence is reached.

In the following two sections, we describe the two optimization procedures in more detail.

\section{A. Network Optimization}

First, let us sketch how to optimize the tensors $\left\{A_{1}, \ldots, A_{M}\right\}$ while keeping the basis fixed. The minimization of the energy with the constraint that the norm remains constant is equivalent to minimizing the functional

$$
F=\langle\Psi|H| \Psi\rangle-E(\langle\Psi \mid \Psi\rangle-1) .
$$

This functional is non-convex with respect to all parameters $\left\{A_{1}, \ldots, A_{M}\right\}$. However, due to the tensor network structure of the ansatz, it is quadratic in the parameters $A_{m}$ associated with one lattice site $m$. Because of this, the optimal parameters $A_{m}$ can simply be found by solving a generalized eigenvalue problem $\mathcal{H}_{m} \vec{A}_{m}=E \mathcal{N}_{m} \vec{A}_{m}$. 
(a)

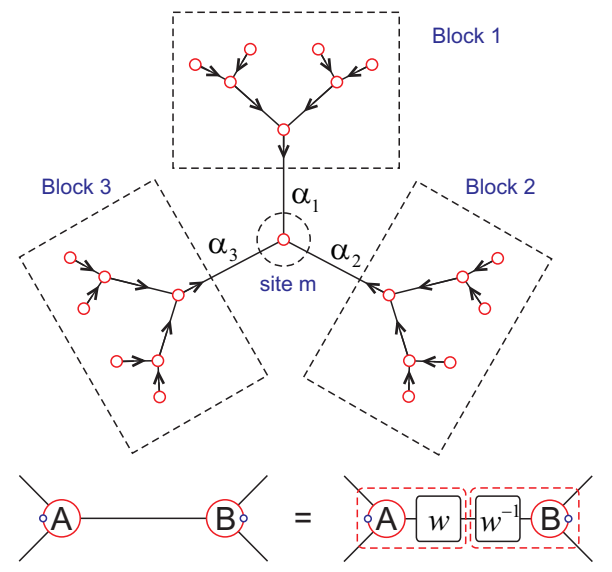

FIG. 2. (Color online) (a) Separation of the state into $z$ blocks plus the site under optimization, as described by Eq. (3). (b) Natural freedom in the tensor network: insertion of a matrix $w$ and $w^{-1}$ at one bond leaves the state invariant. The contraction of $A$ with $w$ forms the new tensor $A^{\prime}$ on the left hand side; the contraction of $B$ with $w^{-1}$ forms the new tensor $B^{\prime}$ at the right hand side.

For a bipartite network, it is always possible to assume a gauge condition so that $\mathcal{N}_{m}=\mathbb{I}$, and thus reduce the generalized eigenvalue problem to an ordinary one ${ }^{33}$. We will discuss this in more detail later in this section. The concept of the algorithm is to do this one-site optimization site-by-site until convergence is reached.

The challenge that remains is to calculate the effective Hamiltonian $\mathcal{H}_{m}$ of the eigenvalue problem. In principle, this is done by contracting all indices in the expression for the expectation value $\langle\Psi|H| \Psi\rangle$ except those that connect to $A_{m}$. By interpreting the tensor $A_{m}$ as a $d D^{z}$ dimensional vector $\vec{A}_{m}$, this expression can be written as

$$
\langle\Psi|H| \Psi\rangle=\vec{A}_{m}^{\dagger} \mathcal{H}_{m} \vec{A}_{m}
$$

Since

$$
\langle\Psi \mid \Psi\rangle=\vec{A}_{m}^{\dagger} \mathcal{N}_{m} \vec{A}_{m}
$$

and $\mathcal{N}_{m}=\mathbb{I}$, the functional $F$ attains its minimum when

$$
\mathcal{H}_{m} \vec{A}_{m}=E \vec{A}_{m}
$$

Due to the bipartite structure of the tensor network, the calculation of $\mathcal{H}_{m}$ can be performed efficiently, i.e., on a time that scales polynomially with $M$ and $D$. Assuming that the coordination number $z$ is the same everywhere, the scaling will be $M d D^{z+1}$.

This procedure is similar to a DMRG calculation with $z$ blocks instead of two, where a block consists of all of the sites within one of the branches emerging from site $m$ (see Fig. 2(a)). The wave function is then formed

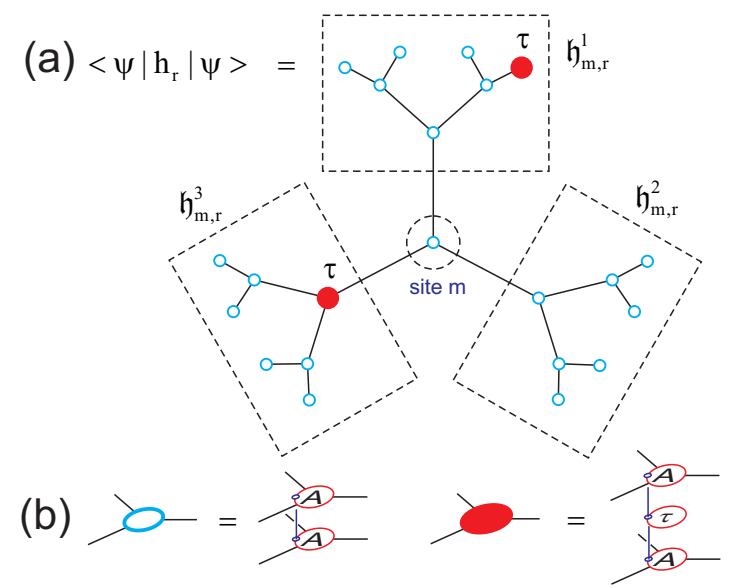

FIG. 3. (Color online) (a) Formation of the effective Hamiltonian $\mathfrak{h}_{m, r}=\mathfrak{h}_{m, r}^{1} \otimes \mathfrak{h}_{m, r}^{2} \otimes \mathfrak{h}_{m, r}^{3}$ with respect to the interaction $h_{r}=\tau^{(7)} \otimes \tau^{(15)}$. The sites on which the interaction has support are marked in red. Each open (filled) circle in the tensor network corresponds to the contraction of the layered structure of tensors shown in (b).

as

$$
|\Psi\rangle=\sum_{\alpha_{1}, \ldots, \alpha_{z}=1}^{D}\left|\varphi_{\alpha_{1} \ldots \alpha_{z}}\right\rangle \otimes\left|\phi_{\alpha_{1}}^{1}\right\rangle \otimes \cdots \otimes\left|\phi_{\alpha_{z}}^{z}\right\rangle,
$$

where $\left|\phi_{\alpha}^{i}\right\rangle(\alpha=1, \ldots, D)$ is the basis in environment block $i(i=1, \ldots, z)$ and $\left|\varphi_{\alpha_{1} \ldots \alpha_{z}}\right\rangle$ is the state of site $m$. Since $\mathcal{N}_{m}$ is obtained from the norm $\langle\Psi \mid \Psi\rangle$ by contracting all tensors except $A_{m}$ [see Eq. (2)], it factorizes into a tensor product of $z$ matrices $\mathcal{N}_{m}^{i}$,

$$
\mathcal{N}_{m}=\mathcal{N}_{m}^{1} \otimes \cdots \otimes \mathcal{N}_{m}^{z},
$$

where each matrix $\mathcal{N}_{m}^{i}$ is formed by taking the overlap of the basis states in environment block $i$ :

$$
\left[\mathcal{N}_{m}^{i}\right]_{\alpha \beta}=\left\langle\phi_{\alpha}^{i} \mid \phi_{\beta}^{i}\right\rangle
$$

Obviously, in order to guarantee that $\mathcal{N}_{m}=\mathbb{I}$, the basis in each environment block must be orthonormal. This is a similar requirement as in the DMRG. In the tree tensor network, this may be achieved by assuming an appropriate gauge condition. The purpose of this gauge condition is to fix the natural freedom in the tensor network that a matrix and its inverse can be inserted at any bond, with the matrix being contracted with the first attached tensor and the inverse being contracted with the second attached tensor, leaving the network invariant (see Fig. 2(b)). The gauge condition for all sites $n$ $(n \neq m)$ that assures that $\mathcal{N}_{m}=\mathbb{I}$ is

$$
\sum_{k \beta_{2} \ldots \beta_{z}}\left[A_{n}^{*}\right]_{\alpha^{\prime} \beta_{2} \ldots \beta_{z}}^{k}\left[A_{n}\right]_{\alpha \beta_{2} \ldots \beta_{z}}^{k}=\delta_{\alpha \alpha^{\prime}} .
$$

Here we take the index $\alpha$ to be outgoing, i.e., the branch attached to this index contains site $m$, and the indices 


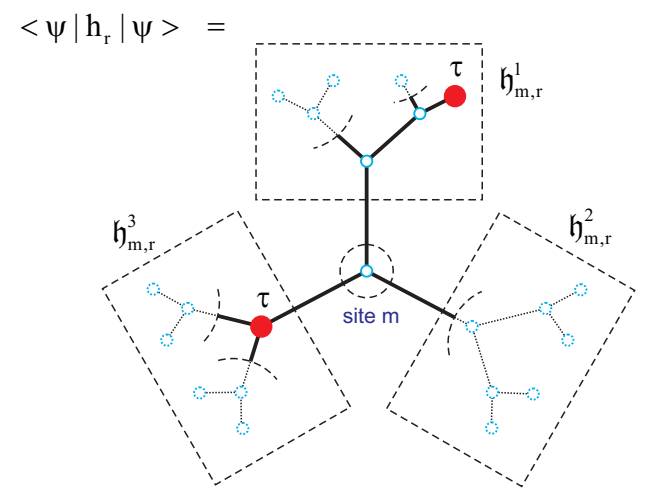

FIG. 4. (Color online) Branches with no support (marked by dotted lines and circles), which yield the identity when contracted and therefore do not have to be taken into account in calculating the effective Hamiltonian.

$\beta_{2} \ldots \beta_{z}$ to be incoming, i.e., the attached branches $d o$ not contain site $m$ (see Fig. 22(a)).

A stable way to apply this gauge condition is to "orthonormalize" the tensors from outside to inside. That is, starting with the tensors on the leaves of the tree, we take into account condition (4) by QR-decomposing $\left[A_{n}\right]_{\alpha}^{k}$, i.e., by writing it as

$$
\left[A_{n}\right]_{\alpha}^{k}=\sum_{\alpha^{\prime}}\left[Q_{n}\right]_{\alpha^{\prime}}^{k} R_{\alpha^{\prime} \alpha}
$$

The unitary matrix $\left[Q_{n}\right]_{\alpha^{\prime}}^{k}$ is the new "orthonormalized" tensor at site $n$. In order to keep the tensor network invariant, $R_{\alpha^{\prime} \alpha}$ must be contracted with the tensor on the first inner shell that is connected to the tensor at site $n$. This procedure continues iteratively with the tensors on the first inner shell, the second inner shell, and so on until site $m$ is reached.

Thus, by assuring that the gauge condition is always satisfied in the course of the algorithm, the only term that must be calculated is the effective Hamiltonian $\mathcal{H}_{m}$. This term is obtained, as can be gathered from Eq. (1), by contracting all tensors except $A_{m}$ in the expectation value $\langle\Psi|H| \Psi\rangle$. Since the Hamiltonian is a sum of interaction terms

$$
H=\sum_{r=1}^{R} h_{r},
$$

with $h_{r}$ being a tensor product of matrices, e.g., $h_{r}=$ $\tau^{(7)} \otimes \tau^{(15)}$ for a two-body interaction acting on sites 7 and 15, the effective Hamiltonian splits into a sum of effective Hamiltonians $\mathfrak{h}_{m, r}$ with respect to a single interaction term $h_{r}$ :

$$
\left\langle\Psi\left|h_{r}\right| \Psi\right\rangle=\vec{A}_{m}^{\dagger} \mathfrak{h}_{m, r} \vec{A}_{m} .
$$

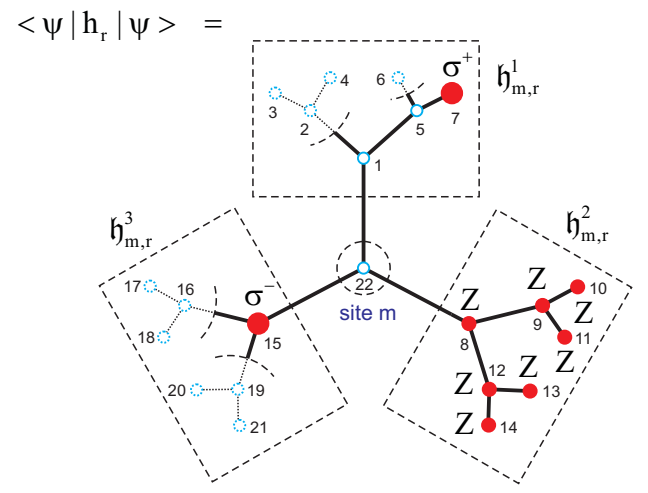

FIG. 5. (Color online) Formation of the effective Hamiltonian $\mathfrak{h}_{m, r}=\mathfrak{h}_{m, r}^{1} \otimes \mathfrak{h}_{m, r}^{2} \otimes \mathfrak{h}_{m, r}^{3}$ with respect to the fermionic interaction $h_{r}=a_{7}^{\dagger} a_{15}$. The sites on which the interaction has support are marked in red.

Due to the structure (3) of the TTNS, each effective Hamiltonian $\mathfrak{h}_{m, r}$ factorizes into a tensor product of $z$ matrices

$$
\mathfrak{h}_{m, r}=\mathfrak{h}_{m, r}^{1} \otimes \cdots \otimes \mathfrak{h}_{m, r}^{z},
$$

where each matrix $\mathfrak{h}_{m, r}^{i}$ corresponds to the matrix elements of $h_{r}$ with respect to the basis in environment block $i$ :

$$
\left[\mathfrak{h}_{m, r}^{i}\right]_{\alpha \beta}=\left\langle\phi_{\alpha}^{i}\left|h_{r}\right| \phi_{\beta}^{i}\right\rangle .
$$

Graphically, the evaluation of $\left\langle\phi_{\alpha}^{i}\left|h_{r}\right| \phi_{\beta}^{i}\right\rangle$ corresponds to the contraction of a three-layered tensor network according to the structure of the branch in block $i$, as depicted in Fig. 3. This network can be contracted efficiently by starting from the leaves and working in the inward direction. The numerical effort for contracting one additional site into the network scales as $d D^{z+1}$, so that the total effort scales as $d D^{z+1}$ times the number of sites in the block.

Of course, if $h_{r}$ has no support in environment block $i$, $\mathfrak{h}_{m, r}^{i}$ is equal to the identity because the basis is chosen to be orthogonal in each environment block. Because of this, the calculation simplifies significantly. For example, each two-site interaction has support in at most two blocks. This means that at most two effective Hamiltonians $\mathfrak{h}_{m, r}^{i}$ have to be calculated (for each interaction term); all others are equal to the identity. Since the orthonormalization of the state is applied iteratively from the leaves inwards to the optimized site $m$, the calculation of $\mathfrak{h}_{m, r}^{i}$ simplifies substantially, as well. Within each block, the contraction of all subbranches on which $\mathfrak{h}_{m, r}^{i}$ has no support automatically yields the identity. Thus, for the example of a two-site interaction, it is sufficient to take into account the sites on the path connecting 
the two sites (see Fig. (4). The treatment of long-range interactions on a tree is therefore not significantly more complicated than the treatment of long-range interaction on a chain in the DMRG.

At first glance, it might seem that the advantage of "cropping" all subbranches with no support is lost when switching to fermions. This is because fermionic interactions with local support are turned into interactions with nonlocal support in the spin picture via the JordanWigner transformation. The two-particle fermionic interaction $a_{7}^{\dagger} a_{15}$, for example, is turned into the spin interaction

$$
a_{7}^{\dagger} a_{15}=\sigma_{+}^{(7)}\left[\prod_{7<n<15} Z^{(n)}\right] \sigma_{-}^{(15)}
$$

that has support on 9 sites, where $Z=-\sigma_{z}$ and $\sigma_{+}, \sigma_{-}$, $\sigma_{z}$ denote the Pauli operators (see Fig. 5). However, as we will show, local fermionic interactions can be treated in the tree network with the same effort as local spin interactions by including the $Z_{2}$-symmetry in the ansatz. This is the fermion number parity in the fermionic language.

The $Z_{2}$-symmetry can be incorporated into the ansatz by making the tensors $\left[A_{m}\right]_{\alpha_{1} \ldots \alpha_{z}}^{k}$ block-diagonal. That is, each virtual index is split into an index pair $\left(\alpha_{i}, p_{i}\right)$, with $p_{i}$ carrying the parity information. By stipulating that $p_{1} \oplus \cdots \oplus p_{z} \oplus k=1$ (where $\oplus$ denotes summation modulo 2), it is possible to "move" the operator $Z$ that acts on the physical index of $A_{m}$ to the virtual parity indices $p_{i}$ :

$$
Z_{k \tilde{k}}\left[A_{m}\right]_{\alpha_{1} p_{1} \cdots \alpha_{z} p_{z}}^{\tilde{k}}=\left[A_{m}\right]_{\alpha_{1} \tilde{p}_{1} \cdots \alpha_{z} \tilde{p}_{z}}^{k} Z_{\tilde{p}_{1} p_{1}} \cdots Z_{\tilde{p}_{z} p_{z}} .
$$

From this relation, it immediatly follows that

$$
Z \otimes \ldots \otimes Z|\Psi\rangle=|\Psi\rangle
$$

and thus that the state is $Z_{2}$-symmetric. This is because the left-hand side of Eq. (6) corresponds to the contraction of $Z$ 's to the physical indices of all tensors $A_{m}$. After moving all $Z$ 's to the virtual bonds, the operator $Z$ appears twice on each bond. Thus, since $Z^{2}=\mathbb{I}$, the state $|\Psi\rangle$ remains unchanged.

Using the same idea, we can immediately enforce a more restrictive symmetry that is fulfilled by all fermionic Hamiltonians: the charge symmetry $U(1)$, i.e. the conservation of the number of particles. For this, the tree graph has to be made directed (see Fig. 6), so that all sites (except one) have $z-1$ incoming and one outgoing bond. The exception is the sink site with $z$ incoming bonds. Thus, each virtual index of a tensor $A_{m}$ is equipped with the additional information of whether it is "incoming" or "outgoing". We assume that the index 1 is always the outgoing index in the following. As before, each virtual index is split into an index pair $\left(\alpha_{i}, n_{i}\right)$. In addition, we require that $n_{1}=n_{2}+\ldots+n_{z}+k$. Thus, for $i=2, \ldots, z, n_{i}$ counts the number of particles within (a)

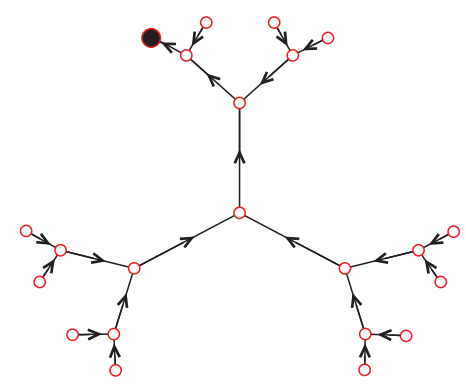

(b)

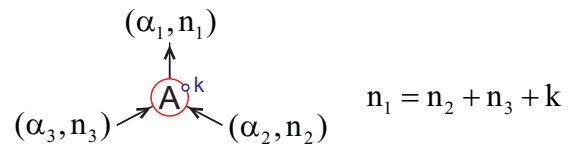

FIG. 6. (Color online) (a) Possible choice of an ordered tree graph with each site having two incoming and one outgoing bond. The sink site is marked in black. The tensors $A_{m}$ associated with each site with virtual indices consisting of pairs $\left(\alpha_{i}, n_{i}\right)$ have the structure shown in (b).

the branch attached to index $i$. The index $n_{1}$, on the other hand, is equal to the number of particles in all the branches plus the number of particles at site $m$. Since the parity can be immediately derived from the particlenumber information, a similar relation as Eq. (5) holds, namely

$$
Z_{k \tilde{k}}\left[A_{m}\right]_{\alpha_{1} n_{1} \cdots \alpha_{z} n_{z}}^{\tilde{k}}=\left[A_{m}\right]_{\alpha_{1} \tilde{n}_{1} \cdots \alpha_{z} \tilde{n}_{z}}^{k} \tilde{Z}_{\tilde{n}_{1} n_{1}} \cdots \tilde{Z}_{\tilde{n}_{z} n_{z}}
$$

with $\tilde{Z}_{\tilde{n} n}=\delta_{\tilde{n} n}(-1)^{n}$. Thus, as before, $Z$ acting on the physical index can be moved to the virtual particlenumber bonds and, since $\tilde{Z}^{2}=\mathbb{I}$, the state is $Z_{2^{-}}$ symmetric.

The advantage of taking into account the particle number is twofold: on the one hand, the block structure of the tensors reduces the numerical effort considerably: a bond-dimension of $\chi=N D$ can be treated with an effort of order $N^{z} D^{z+1}$ instead of $\chi^{z+1}$ for a non-symmetric ansatz. Here $\chi$ is the full bond dimension, so it is equivalent to the number of block states kept in a DMRG procedure. On the other hand, the calculation of the effective Hamiltonians $\mathfrak{h}_{m, r}$ with respect to an interaction with support on a few sites only, e.g., a twoparticle or four-particle interaction, is simplified. The main idea is depicted in Fig. 7 for the interaction $a_{7}^{\dagger} a_{15}$ : with an appropriately chosen numbering of the fermions, each subbranch that has no fermionic support either has only identities acting on the sites or only operators $Z$. The subbranches including only identities simplify to the identity because of the orthonormalization of the state. The $Z$ operators can be moved, using relation (7), to the virtual bonds, and all of them except one cancel (see Figs. 5 and (7). What remains is a subbranch that includes only identities, which reduces to the identity because of the orthonormalization of the state. Thus, for a 


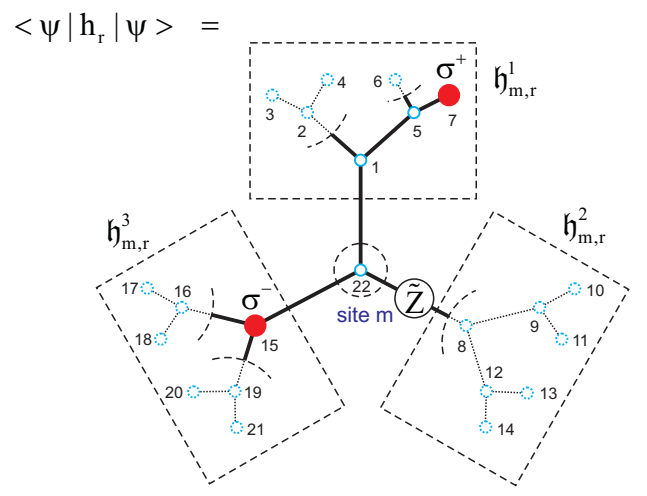

FIG. 7. (Color online) Formation of the effective Hamiltonian $\mathfrak{h}_{m, r}=\mathfrak{h}_{m, r}^{1} \otimes \mathfrak{h}_{m, r}^{2} \otimes \mathfrak{h}_{m, r}^{3}$ with respect to the fermionic interaction $h_{r}=a_{7}^{\dagger} a_{15}$ with parity symmetry taken into account. The sites on which the interaction has support are marked in red. All branches marked by dotted lines and circles yield the identity when contracted. The parity operator $\tilde{Z}$ is contracted to the virtual bond.

fermionic two-site interaction, it is sufficient to take into account the path connecting the two sites. In this way, the treatment of long-range fermionic interactions is feasible with the same numerical effort as the treatment of long-range spin interactions.

Using the same scheme as for minimizing the energy, the efficient simulation of time evolution is also possible. For this, the time evolution is split up into small steps of duration $\delta t$. For $\delta t \ll 1$ and a starting state $\left|\Psi_{0}\right\rangle$ of the form of a TTNS, the time-evolved state $e^{-i H \delta t}\left|\Psi_{0}\right\rangle$ is a TTNS as well. However, the virtual dimension is multiplied by a factor $\kappa>1$. In order to prevent the bond dimension from increasing exponentially with time, the TTNS has to be approximated by a TTNS with a reduced virtual dimension $|\Psi\rangle$ after every time step, i.e., the functional

$$
K=\| e^{-i H t}\left|\Psi_{0}\right\rangle-|\Psi\rangle \|^{2}
$$

must be minimized. The optimization with respect to a single site $m$ leads to the system of linear equations $\mathcal{N}_{m} \vec{A}_{m}=\vec{w}_{m}$ (where $\mathcal{N}_{m}$ can again be set equal to identity by using the appropriate gauge) with

$$
\left\langle\Psi\left|e^{-i H t}\right| \Psi_{0}\right\rangle=\vec{A}_{m}^{\dagger} \vec{w}_{m}
$$

and thus can be performed efficiently with the scaling $M D^{z+1}$, as before. Clearly, all previous considerations regarding particle-number symmetry can also be adapted to the case of time evolution.

\section{B. Orbital Optimization}

As mentioned previously, the entanglement properties of the system depend critically on the choice of the basis. Our goal is to find a basis in which entanglement is localized as much as possible at the sites of the tree network. Such a choice of basis guarantees that a given precision can be attained with a smaller virtual dimension $D$, and thus with less computational effort. In QC-DMRG the optimization of the basis is fundamental and has been used in much previous work $32,51-54$

In contrast to previous work, our approach aims to find the optimal basis that can be obtained by a canonical transformation of the fermionic modes. The canonical transformation is defined by a $M \times M$ unitary matrix $U$. Since the number of parameters is relatively small, a gradient search applied to the expectation value of the energy,

$$
E(U)=\langle\Psi(U)|H| \Psi(U)\rangle
$$

is certainly feasible. Since $E(U)$ is a non-convex function of the parameters $U$, it is a highly non-trivial problem to find the absolute minimum. The idea is to perform the gradient search multiple times in the course of the algorithm, e.g., after each optimization sweep of the tensor network, and improve the energy at each gradient search by only a small amount. This will eventually adapt the orbitals optimally to the tree tensor network.

There are two ways to implement the basis transformation: one based on the state and the other based on the Hamiltonian. We have applied the basis transformation to the Hamiltonian. For the fermionic Hamiltonian with long-range interactions,

$$
H=\sum_{i j} T_{i j} a_{i}^{\dagger} a_{j}+\sum_{i j k l} V_{i j k l} a_{i}^{\dagger} a_{j}^{\dagger} a_{k} a_{l},
$$

that appears, e.g., in quantum chemistry, in momentumspace descriptions of the Hubbard model, or in descriptions of the Hubbard model on higher dimensional lattices, the function $E(U)$ can be be expressed as

$$
E(U)=\sum_{i j} \tilde{T}(U)_{i j}\left\langle a_{i}^{\dagger} a_{j}\right\rangle+\sum_{i j k l} \tilde{V}(U)_{i j k l}\left\langle a_{i}^{\dagger} a_{j}^{\dagger} a_{k} a_{l}\right\rangle
$$

with

$$
\begin{aligned}
& \tilde{T}(U)=U T U^{\dagger} \\
& \tilde{V}(U)=(U \otimes U) V(U \otimes U)^{\dagger} .
\end{aligned}
$$

The correlation functions $\left\langle a_{i}^{\dagger} a_{j}\right\rangle$ and $\left\langle a_{i}^{\dagger} a_{j}^{\dagger} a_{k} a_{l}\right\rangle$ are calculated with respect to the original state and are not dependent on the parameters in $U$. With the function $E(U)$ in this form, its gradient can be calculated explicitly. Both quantities can be evaluated efficiently for different parameter sets $U$, which makes the gradient search feasible.

The gradient search that is used for the orbital optimization typically finds the local minima in the vicinity 

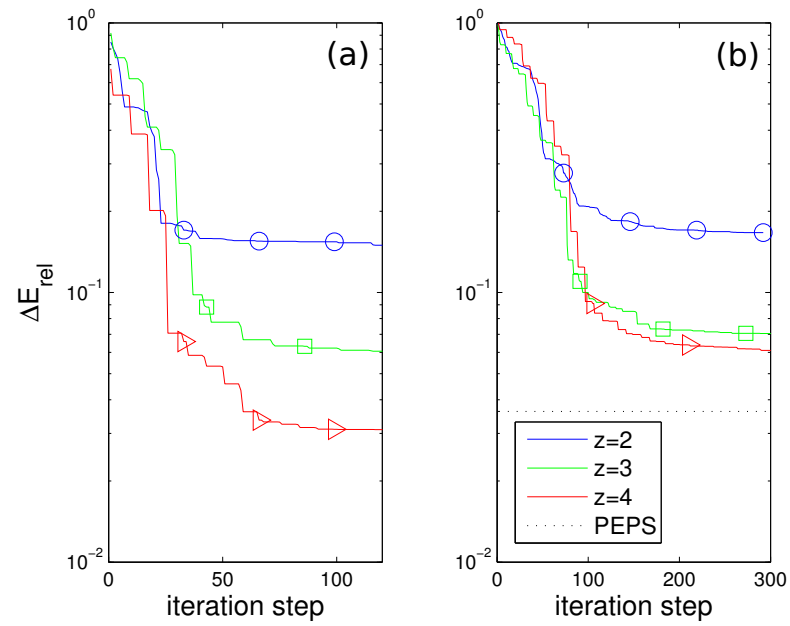

FIG. 8. (Color online) Relative error in the energy for Heisenberg model on a $4 \times 4$-lattice (a) and a $6 \times 6$-lattice (b) as a function of the optimization steps. The calculations were performed with a fixed virtual dimension of $D=4$ and tree tensor networks with coordination numbers $z=2$ (blue curve), $z=3$ (green curve) and $z=4$ (red curve).

of the starting point of the optimization. In order to avoid falling into the same minimum, we shift the starting point in a random direction by an appropriately chosen amount. This has the consequence that the gradient search generally falls into another local minimum that may have a higher energy. However, it assures that the orbitals change considerably and that, since the orbital optimization is performed repeatedly interspersed with network optimization, the energy decreases notably in the course of the algorithm.

\section{NUMERICAL RESULTS}

We have applied the algorithm consisting of two optimization procedures described above, the optimization of the tensor network and the optimization of the basis according to this network, to several models. In this section, we discuss the results.

\section{A. 2-d Heisenberg model}

First, let us consider the tree tensor network optimization only and show using the 2-d Heisenberg model how results improve with a TTNS ansatz compared to a onedimensional MPS ansatz. The relative error in the energy of a system consisting of $4 \times 4$ spins as a function of the optimization step is shown in the left hand side of Fig. 8 for the MPS ansatz and the TTNS ansatz with $z=3$ and $z=4$. In all calculations, a fixed virtual dimension of $D=4$ is used. We have assigned a spin to each node in
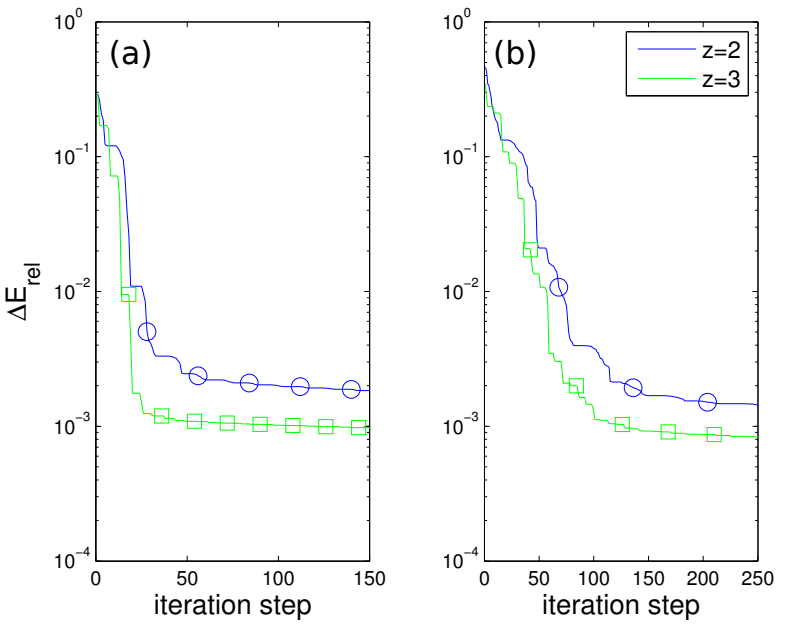

FIG. 9. (Color online) Relative error in the energy for the interacting spinless fermion model on (a) a $4 \times 4$-lattice and (b) a $6 \times 6$-lattice as a function of the optimization steps. The calculations were performed with a fixed virtual dimension of $D=4$ and tree tensor networks with coordination numbers $z=2$ (blue curve) and $z=3$ (green curve). The chosen parameters are $J=1, U=0.5$ and the number of fermions is fixed to $N=3$.

the tree network in such a way that two arbitrary spins are connected by the smallest possible number of bonds. As can be seen, the precision increases considerably with increasing coordination number.

For larger systems such as for $6 \times 6$ spins, shown in the right hand side of Fig. 8, the energy is plotted as a function of the optimization steps for $z=2, z=3$, and $z=4$. The virtual dimension is fixed to $D=4$. As before, the energy decreases considerably and approaches the PEPS result for $D=4$, indicated by the dotted line which, however, is obtained with a much larger numerical effort. 25

\section{B. 2-d interacting spinless fermions}

The TTNS Ansatz also perfoms well on twodimensional fermionic models. We have applied the TTNS algorithm to a system of interacting fermions on a two-dimensional lattice, described by the Hamiltonian

$$
H=-J \sum_{<i, j>} a_{i}^{\dagger} a_{j}+U \sum_{<i, j>} \hat{n}_{i} \hat{n}_{j}
$$

with $\hat{n}_{i}=a_{i}^{\dagger} a_{i}$. The boundary conditions are assumed to be periodic. As can be gathered from Fig. 9, the groundstate energy improves with increasing coordination number $z$. The figure shows the relative error in the energy as a function of the iteration step for $z=2$ and $z=3$ for a $4 \times 4$-lattice in the left part, and the ground state energy as a function of the iteration step for a $6 \times 6$-lattice in the 

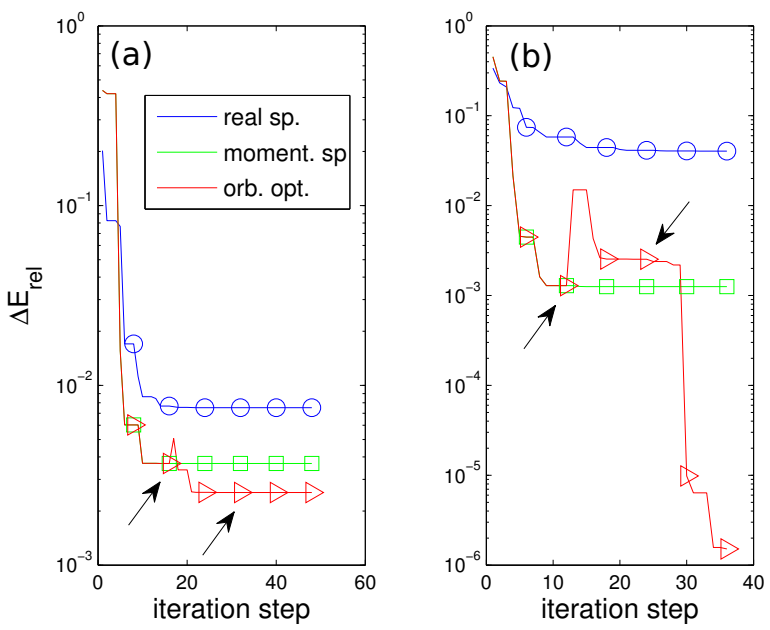

FIG. 10. (Color online) Relative error in the energy for interacting spinless fermions with $M=7$ sites as a function of the optimization steps for the TTNS ansatz with $z=2$ (a) and $z=3$ (b). Here $U=1$, the particle number is fixed to $N=3$, and the virtual dimension is assumed to be $D=2$. Results with orbital optimization are compared to the results obtained in the real-space basis and in the momentum-space basis. The steps at which orbital optimizations are performed are marked with arrows.

right part. For the calculations, we have used the virtual dimension $D=4$. We have chosen the parameters $J=1$ and $U=0.5$ and have fixed the number of fermions to $N=3$.

Thus, a TTNS Ansatz might be useful for the study of higher dimensional models of small size because the effective long-range interactions are represented better in a tree than in a chain and the numerical effort is relatively low compared to a PEPS calculation.

\section{1-d interacting spinless fermions}

In order to assess the effectiveness of the orbital optimization, we have applied the algorithm to a simple fermionic model, the one-dimensional interacting spinless fermion model

$$
H=-J \sum_{i=1}^{M}\left(a_{i}^{\dagger} a_{i+1}+\text { h.c. }\right)+U \sum_{i=1}^{M} \hat{n}_{i} \hat{n}_{i+1},
$$

where $\hat{n}_{i}=a_{i}^{\dagger} a_{i}$. We assume periodic boundary conditions, i.e., $a_{M+1}=a_{1}$. This model can be mapped to the XXZ spin chain via a Jordan-Wigner transformation. In this model, it is known that the choice of the basis has a big effect on the precision of the DMRG or TTNS calculation. For $U \rightarrow \infty$, the ground state is a product state in the position representation, and thus optimally represented by a tensor network with $D=1$ in this basis. For $U=0$, on the other hand, the ground state can

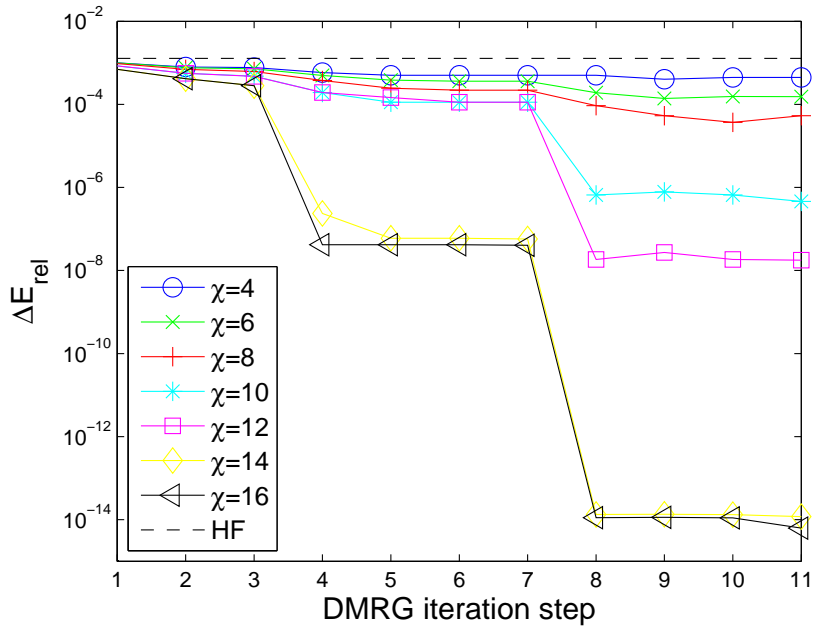

FIG. 11. (Color online) The relative error in the groundstate energy for the Beryllium atom as a function of DMRG iteration steps for various values of the number of DMRG block states. The dashed line corresponds to the HartreeFock energy.

be represented as a direct product in momentum space. Thus, the momentum-space basis is clearly best suited in this limit. For intermediate $U$, one might expect a basis intermediate between real and momentum space to represent the entanglement properties optimally; our goal is to find such a basis automatically by carrying out orbital optimization.

The results of calculations incorporating the optimization procedure are displayed in Fig. 10 for $z=2$ and $z=3$, both on systems of $M=7$ sites and $N=3$ particles with a fixed virtual dimension of $D=2$ and parameters $U=1$. As before, the relative error in the energy as a function of the optimization step is plotted. For comparison, calculations performed in the position representation and in the momentum representation are also shown. As can be seen, the energy improves significantly in the course of the optimization; the improvement is three orders of magnitude for the $z=3$ case.

\section{Quantum Chemical systems}

In this section, we compare numerical results for quantum chemical systems obtained using the QC-DMRG and TTNS methods. In these applications, the electronelectron correlation is taken into account by an iterative procedure that minimizes the Rayleigh quotient corresponding to the Hamiltonian describing the electronic structure of the molecule, given by

$$
H=\sum_{i j \sigma} T_{i j} a_{i \sigma}^{\dagger} a_{j \sigma}+\sum_{i j k l \sigma \sigma^{\prime}} V_{i j k l} a_{i \sigma}^{\dagger} a_{j \sigma^{\prime}}^{\dagger} a_{k \sigma^{\prime}} a_{l \sigma}
$$




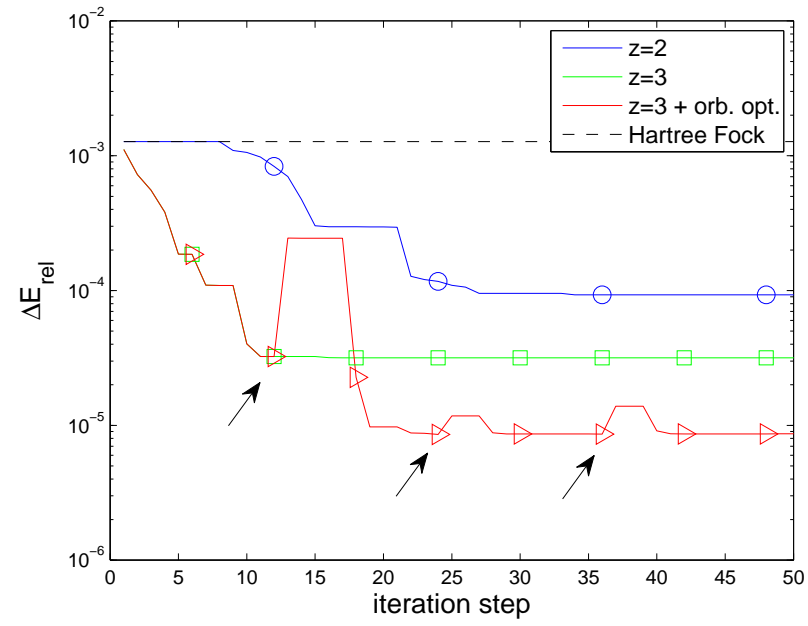

FIG. 12. (Color online) Relative error in the ground-state energy for the Be atom as a function of the optimization step for $D=2$. The results obtained using a TTNS ansatz with $z=3$ plus orbital optimization are compared to the TTNS-results without orbital optimization For comparison, the results with a MPS ansatz (corresponding to $z=2$ ) are also included. The steps at which orbital optimizations are performed are marked with arrows.

and thus determines the full-CI wave function. In Eq. (8), $T_{i j}$ denotes the matrix elements of the oneparticle Hamiltonian, which is comprised of the kinetic energy and the external electric field of the nuclei, and $V_{i j k l}$ stands for the matrix elements of the electron repulsion operator, defined as

$$
V_{i j k l}=\int d^{3} x_{1} d^{3} x_{2} \Phi_{i}^{*}\left(\vec{x}_{1}\right) \Phi_{j}^{*}\left(\vec{x}_{2}\right) \frac{1}{\vec{x}_{1}-\vec{x}_{2}} \Phi_{k}\left(\vec{x}_{2}\right) \Phi_{l}\left(\vec{x}_{1}\right) .
$$

We obtain the Hartree-Fock orbitals in a given basis of Gaussian orbitals and transform the matrix elements $T_{i j}$ and $V_{i j k l}$ to the Hartree-Fock basis using the MOLPRO program package,,$\frac{55}{5}$ wich we also use to obtain the fullCI energies used as a benchmark.

In the QC-DMRG, a one-dimensional chain is built up from the atomic or molecular orbitals obtained from a suitable mean-field or MCSCF calculation. The tree network is constructed similarly, but there is greater freedom to form the proper structure of the network. The two-orbital mutual information ${ }^{15}$ provides a good starting configuration. A general approach to reduce entanglement is to form the network by placing the highly correlated orbitals at or near the center of the tree and less correlated orbitals at the boundary sites.

In Fig. 11 we plot the relative error in the ground-state energy for the Beryllium atom as a function of DMRG iteration steps for various fixed values of the DMRG block states. Corresponding data gathered after the fourth DMRG sweep is shown in Fig. 13. In this calculation, four electrons have been placed on eight orbitals. The
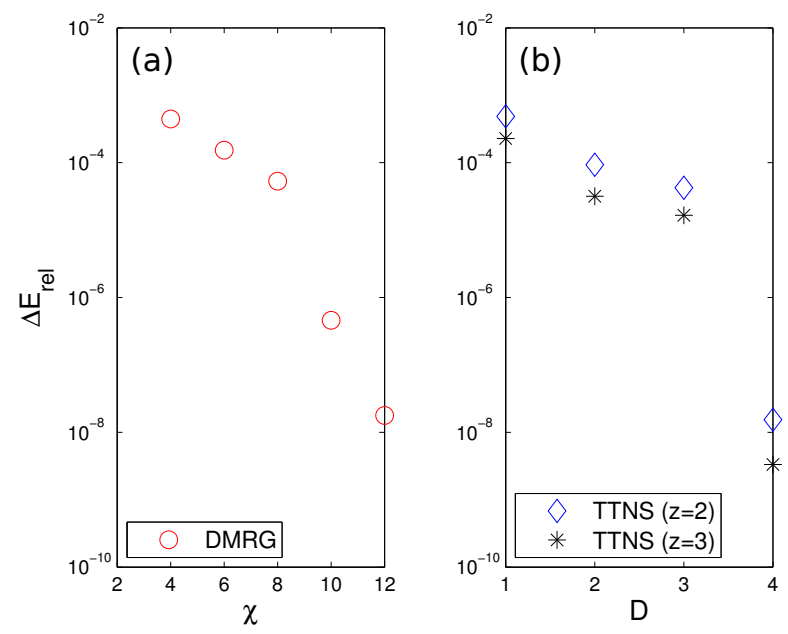

FIG. 13. (Color online) Relative error in the ground-state energy for the Beryllium atom as a function of the full bond dimension $\chi$ obtained using (a) the DMRG and (b) as a function of the virtual dimension $D$ obtained from a TTNS with $z=2$ and $z=3$.

Hartree-Fock energy is -14.351880250000 , while the fullCI energy is -14.37016558404629 . Figure. 12 depicts the relative error as a function of the optimization step for the Be atom, calculated using a TTNS ansatz with coordination number $z=3$. The cases with and without orbital optimization are considered separately, and the $z=2$ results are included for comparison. As can be seen, the relative error is considerably smaller for $z=3$ than for $z=2$, and there is a significant gain in precision when orbital optimization is taken into account. In Fig. 13. we display the dependence of the $z=2$ and 3 calculations on bond dimension $D$. As can be seen, the DMRG and TTNS calculations with $z=2$ yield similar accuracy, while the $z=3$ TTNS calculation is significantly more accurate for a given bond dimension.

It should be noted that there is currently a discrepancy between the QC-DMRG and the TTNS calculations in the speed of convergence with optimization step. Since our QC-DMRG code is highly optimized, the calculation converges faster than that in the present version of the TTNS method. As can be seen in Fig. 11, for example, the QC-DMRG ground-state energies are always below the Hartree-Fock energy, and the active space is extended dynamically using CI-based expansion techniques (CIDEAS $)^{\frac{5}{2}}$ (DEAS). In general, there is no fundamental difficulty in incorporating optimizations such as the CIDEAS into the TTNS method.

\section{CONCLUSION}

In this paper, we have described and applied a method, the tree tensor network state method, to treat strongly 
correlated systems with long-range interactions on a tree network with arbitrary coordination number $z$. Our approach is based on a tensor product state ansatz that generalizes a DMRG-like matrix product state to $z$ rather than two blocks. The number of virtual bonds required to connect two arbitrary sites scales logarithmically with the number of sites in TTNS, in contrast to the linear scaling of a one-dimensional topology ${ }^{33}$ In this sense, our TTNS method has a lower computational cost than currently used DMRG-based methods. We have also incorporated optimization of the single-particle orbitals in our method, treating the case of a procedure for tranforming the basis that smoothly interpolates between real space and momentum space. We have tested our method using numerical calculations on various systems with local and nonlocal interactions, including the two-dimensional Heisenberg lattice, the momentum-space version of the 1$\mathrm{d}$ interacting spinless fermion model, and small quantum chemical systems. For the quantem chemical systems, we have compared TTNS results to those of DMRG calculations.

Since the TTNS approach is defined on a bipartite network, previous algorithmic developments and optimizations procedures developed in the context of the quantum chemistry version of DMRG can also be integrated into the TTNS method. Such optimizations include dynamic adjustment of the bond dimension, $\frac{5}{-}$ orbital optimization, $\stackrel{32,51-54,56,57}{5}$ and initialization procedures based on CI expansions, $11,14,58$ among others. Incorporation of these elements into the TTNS approach will be carried out in future work. In light of the promising features of the new method, we expect it to provide a viable alternate means of treating atoms and molecules efficiently in the near future.

\section{ACKNOWLEDGMENTS}

We thank I. Cirac, N. Schuch, and S. R. White for very valuable discussions. V. M. and F. V. acknowledge support from the SFB projects FoQuS and ViCoM, the European projects Quevadis, and the ERC grant Querg. O. L. acknowledges support from the Alexander von Humboldt foundation and from the Hungarian Research Fund(OTKA) through Grant Nos. K68340 and K73455. We would especially like to thank the ErwinSchrödinger-Institut in Vienna for its hospitality during the Quantum Computation and Quantum Spin Systems workshop in 2009, where many fruitful discussion took place.
* Also at Research Institute for Solid-State Physics and Optics, Hungarian Academy of Sciences, H-1121 Budapest, Hungary

1 S. R. White, Phys. Rev. Lett 69, 2863 (1992).

2 S. R. White, Phys. Rev. B 48, 10345 (1992).

3 T. Xiang, Phys. Rev. B 53, R10445 (1996).

4 S. Nishimoto, E. Jeckelmann, F. Gebhard, and R. M. Noack, Phys. Rev. B 65, 165114 (2002).

${ }^{5}$ Ö. Legeza and J. Solyom, Phys. Rev. B 68, 195116 (2003).

6 S. R. White and R. L. Martin, J. Chem. Phys. 110, 4127 (1999).

7 S. Daul, I. Ciofini, C. Daul, and S. R. White, Int. J. Quantum Chem. 79, 331 (2000).

8 A. O. Mitrushenkov, G. Fano, F. Ortolani, R. Linguerri, and P. Palmieri, J. Chem. Phys. 115, 6815 (2001).

${ }^{9}$ G. K.-L. Chan and M. Head-Gordon, J. Chem. Phys. 116, 4462 (2002).

10 G. K.-L. Chan and M. Head-Gordon, J. Chem. Phys. 118, 8551 (2003).

11 Ö. Legeza, J. Röder, and B. A. Hess, Phys. Rev. B 67, 125114 (2003), cond-mat/0204602.

12 Ö. Legeza, J. Röder, and B. A. Hess, Mol. Phys. 101, 2019 (2003), cond-mat/0208187.

13 G. Moritz, B. A. Hess, and M. Reiher, J. Chem. Phys. 122, 024107 (2005).

14 G. Moritz, B. A. Hess, and M. Reiher, J. Chem. Phys. 124, 034103 (2006).

15 J. Rissler, R. M. Noack, and S. R. White, Chem. Phys. 323, 519 (2006), cond-mat/0508524.

16 K. Marti, I. M. Ondik, G. Moritz, and M. Reiher, J. Chem. Phys. 128, 014104 (2008).

17 G. Vidal, J. I. Latorre, E. Rico, and A. Kitaev, Phys. Rev.
Lett 90, 227902 (2003), quant-ph/0211074.

18 G. Vidal, Phys. Rev. Lett 91, 147902 (2003), quant-ph/0310089.

19 O. Legeza and J. Solyom, Phys. Rev. B 70, 205118 (2004).

20 F. Verstraete, J. I. Cirac, and V. Murg, Adv. Phys. 57 (2), 143 (2008).

21 F. Verstraete and J. Cirac, Phys. Rev. B 73, 094423 (2006), cond-mat/0505140.

22 N. Schuch, M. M. Wolf, F. Verstraete, and J. I. Cirac, Phys. Rev. Lett. 100, 030504 (2008).

23 S. Östlund and S. Rommer, Phys. Rev. Lett. 75, 3537 (1995).

24 F. Verstraete and J. I. Cirac, arXiv:condmat/0407066v1(2004).

25 V. Murg, F. Verstraete, and J. I. Cirac, Phys. Rev. A 75, 033605 (2007), cond-mat/0611522.

${ }^{26}$ V. Murg, F. Verstraete, and J. I. Cirac, Phys. Rev. B 79, 195119 (2009).

27 G. Vidal, Phys. Rev. Lett. 101, 110501 (2008).

28 H. J. Changlani, J. M. Kinder, C. J. Umrigar, and G. K.-L. Chan, arXiv:0907.4646v3 [cond-mat.str-el](2009).

29 K. H. Marti, B. Bauer, M. Reiher, M. Troyer, and F. Verstraete, arXiv:1004.5303v1 [physics.chem-ph](2010).

30 D. M. Ceperley and B. J. Alder, Phys. Rev. Lett. 45, 566 (1980).

31 M. Troyer and U. Wiese, Phys.Rev.Lett. 94, 170201 (2005), cond-mat/0408370

32 H. G. Luo, M. P. Qin, and T. Xiang, arXiv:1002.1287v1 [cond-mat.str-el] (2010).

33 Y. Shi, L. Duan, and G. Vidal, Phys. Rev. A 74, 022320 (2006).

34 U. Schollwöck, Rev. Mod. Phys. 77, 259 (2005), 
cond-mat/0409292

35 M. A. Cazalilla and J. B. Marston, Phys. Rev. Lett. 88, 256403 (2002).

36 S. R. White and A. E. Feiguin, Phys. Rev. Lett 93, 076401 (2004), cond-mat/0403310.

37 A. J. Daley, C. Kollath, U. Schollwoeck, and G. Vidal, J. Stat. Mech.: Theor. Exp., P04005 (2004), cond-mat/0403313.

38 K. G. Wilson, Rev. Mod. Phys. 47, 773 (1975).

39 W. Hofstetter, Phys. Rev. Lett. 85, 1508 (2000).

${ }^{40}$ R. Peters, T. Pruschke, and F. B. Anders, Phys. Rev. B 74, 245114 (2006).

41 F. B. Anders and A. Schiller, Phys. Rev. Lett. 95, 196801 (2005).

42 A. I. Toth, C. P. Moca, Ö. Legeza, and G. Zarand, Phys. Rev. B 78, 245109 (2008).

43 A. Weichselbaum and J. von Delft, Phys. Rev. Lett. 99, 076402 (2007).

44 A. Holzner, A. Weichselbaum, and J. von Delft, Phys. Rev. B 81, 125126 (2010).

45 A. Georges, G. Kotliar, W. Krauth, and M. J. Rozenberg, Rev. Mod. Phys. 68, 13 (1996).

46 M. Eckstein, M. Kollar, K. Byczuk, and D. Vollhardt, Phys. Rev. B 71, 235119 (2005).

47 H. Otsuka, Phys. Rev. B 53, 14004 (1996).

48 B. Friedman, J. Phys.: Condens. Matter 9, 9021 (1997).

49 M. B. Lepetit, M. Cousy, and G. Pastor, Eur. Phys. J. B
13, 421 (2000).

50 L. Tagliacozzo, G. Evenbly, and G. Vidal, Phys. Rev. B 80, 235127 (2009).

51 S. R. White, J. Chem. Phys. 117, 7472 (2002).

52 D. Zgid and M. Nooijen, J. Chem. Phys. 128, 144116 (2008).

53 D. Zgid and M. Nooijen, J. Chem. Phys. 128, 014107 (2008).

54 D. Ghosh, J. Hachmann, T. Yanai, and G. K.-L. Chan, J. Chem. Phys. 128, 144117 (2008).

55 R. D. Amos, A. Bernhardsson, A. Berning, P. Celani, D. L. Cooper, M. J. O. Deegan, A. J. Dobbyn, F. Eckert, C. Hampel, G. Hetzer, P. J. Knowles, T. Korona, R. Lindh, A. W. Lloyd, S. J. McNicholas, F. R. Manby, W. Meyer, M. E. Mura, A. Nicklass, P. Palmieri, R. Pitzer, G. Rauhut, M. Schütz, U. Schumann, H. Stoll, A. J. Stone, R. Tarroni, T. Thorsteinsson, and H. J. Werner, MOLPRO, a package of ab initio programs designed by H.-J. Werner and P. J. Knowles, version 2002.1.

56 T. Yanai and G. K.-L. Chan, J. Chem. Phys. 124, 194106 (2006).

57 T. Yanai, Y. Kurashige, E. Neuscamman, and G. K.-L. Chan, J. Chem. Phys. 132, 024105 (2010).

58 G. Moritz and M. Reiher, J. Chem. Phys. 126, 244109 (2007). 